\title{
La Extensión Universibaria como herramienta de generación de políticas públicas para las personas con discapacidad
}

C. Copyright 2021. Universidad Nacional Autónoma de Nicaragua, Managua (UNAN-Managua) Todos los derechos reservados

\section{University Extension as a tool for generating public policies for people with disabilities}

Odderey José Matus Gómez

https://orcid.org/0000-0003-0040-5190

odderey.matus@gmail.com

\section{César Alejandro Otero Escorcia}

https://orcid.org/0000-0001-9058-9434

birmaniazamora@gmail.com

Fecha de recibido: 25/04/2021

\section{Resumen}

Los problemas que afrontan las personas con discapacidad han sido considerados, a nivel internacional, como temas de mayor atención en los últimos tiempos. Las instituciones de educación superior, especialmente las de carácter público, han de tomar beligerancia en asumir estos desafíos a través de la función de Extensión, la cual debe convertirse en herramienta clave para la visibilización de estos problemas, especialmente con acciones formales como protocolos, campañas de sensibilización, y finalmente, la aprobación de políticas de extensión universitaria, a fin de generar propuestas sólidas que puedan llegar a convertirse en políticas públicas efectivas, que deban ser asumidas y promovidas por los respectivos Estados.

\section{Palabras claves}

Extensión universitaria, políticas públicas, personas con discapacidad, metodologías.
Bertha María Conto Laínez

https://orcid.org/0000-0001-5148-7857

berthaconto@gmail.com

Birmania Zamora Arrechavala

https://orcid.org/0000-0002-5298-2240

otero.30@gmail.com

Fecha de dictaminado: 31/05/2021

\section{Summary}

The problems faced by people with disabilities have been considered, at the international level, as issues of greater attention in recent times. Higher education institutions, especially those of a public nature, must be belligerent in assuming these challenges through the Outreach function, which should become a key tool for making these problems visible, especially with formal actions such as protocols, campaigns of awareness, and finally the approval of university extension policies, in order to generate solid proposals that can become effective public policies that must be assumed and promoted by the respective States.

\section{Keywords}

University extension, public policies, people with disabilities, methodology. 


\section{Introducción}

En el siguiente ensayo titulado "La Extensión Universitaria como herramienta de generación de políticas públicas para las personas con discapacidad", se pretende hacer una reflexión en torno al papel que debe jugar la extensión universitaria respecto a las personas con discapacidad. Metodológicamente, para este ensayo se ha empleado en primer lugar la técnica de análisis documental. Esta técnica, incluye varias etapas que son: el rastreo e inventario, clasificación y selección de los documentos pertinentes para el ensayo y al finalizar la lectura a profundidad de los documentos utilizados. Como segunda, se ha aplicado una breve encuesta, dirigida a una muestra intencional de funcionarios de extensión de universidades miembros del Consejo Nacional de Universidades de Nicaragua (CNU), a la que respondieron siete de diez seleccionados, a fin de consultar sus "estados de opinión o diversas cuestiones de hechos" (DRAE, 2021), sobre el tipo de discapacidades detectadas en la comunidad educativa, estrategias de atención, experiencias y sugerencias para atender la situación.

Este ensayo, es un fruto colectivo de trabajo final del Módulo V: "Políticas, Sistemas y Culturas Escolares", del Doctorado de Educación e Intervención Social (quinta edición) de UNAN-Managua, el cual inicia con una reflexión sobre la atención internacional que ha cobrado este tema en las últimas décadas desde el escenario de las naciones unidas, a fin de comprender el fundamento en que se apoyan las principales leyes que se han generado en el contexto nicaragüense sobre el problema. Seguidamente, se delimita aún más el discurso al exponer algunos referentes sobre el abordaje del tema desde la educación superior nacional y el aporte que esta tiene de frente al análisis que debe realizar para la generación de política públicas.

Finalmente, se expone brevemente el sentido de la extensión universitaria como herramienta que permita visibilizar el problema, en función de generar políticas públicas efectivas, aspecto en el cual se centra el objetivo clave de esta reflexión, así como las experiencias acometidas por la Universidades del $\mathrm{CNU}$ en la práctica. Se concluye con la presentación de algunas ideas en torno a cómo se puede vincular la extensión universitaria en el ejercicio analítico de políticas públicas para atender a las personas con discapacidad, sobre la base de las orientaciones generales que se han brindado desde los foros internacionales y las experiencias locales en nuestro país.

\section{Reflexión en torno a la extensión universitaria y las personas con discapacidad}

\section{La personas con discapacidad como parte de la agenda internacional}

Una de las principales iniciativas que han centrado su atención sobre las personas con discapacidad a nivel mundial, es la Convención Internacional sobre los Derechos de las Personas con Discapacidad, promulgada por las Naciones Unidas el 13 de diciembre de 2006, para promover, proteger y garantizar los derechos humanos de las personas con discapacidad y garantizar el goce pleno de igualdad ante la ley. En el Arto 1 de la Declaración, los Estados firmantes reconocen que:

Las personas con discapacidad incluyen a aquellas que tengan deficiencias físicas, mentales, intelectuales o sensoriales a largo plazo que, al interactuar con diversas barreras, puedan impedir su participación plena y efectiva en la sociedad, en igualdad de condiciones con las demás.

En dicha convención y su protocolo facultativo, se afirma que las personas con discapacidad deben gozar de todos los derechos humanos y las libertades fundamentales. También, incluyen un marco referencial respecto a la promulgación, supervisión de normas mínimas y directrices sobre la accesibilidad, el desarrollo de instalaciones que eliminen las barreras de movilidad, comunicación y libertad de las personas con discapacidad, y que están directamente relacionadas con el quehacer de los centros educativos, como colegios y universidades, a los cuales estas personas tienen el derecho a acceder. En su artículo 24, la Convención reconoce el derecho a las personas con discapacidad a la educación. Propone un sistema educativo inclusivo a todos los niveles, así como la enseñanza a lo largo de toda la vida. El desarrollo pleno de las personas es el fin último de la convención, promoviendo habilidades para la vida y el desarrollo social.

Otras iniciativas que incluyen el tema de las personas con discapacidad, surgen en el año 2015, cuando los líderes mundiales adoptaron nuevos objetivos globales para erradicar la pobreza, proteger el planeta y asegurar la prosperidad para todos: Los Objetivos de Desarrollo Sostenible (ODS), de la Agenda 2030. Dichos objetivos proponen entre una diversidad de acciones, el evitar la exclusión social de las personas con discapacidad y 
asegurarles una vida digna y un empleo seguro. Entre los ODS enfocados en el desarrollo de las personas con discapacidad sobresalen los siguientes cinco:

- Objetivo 4: donde se propone el acceso igualitario a todos los niveles de la enseñanza y la formación profesional. Así como la creación o habilitación de instalaciones educativas que tengan en cuenta las necesidades de las personas con discapacidad.

- Objetivo 8: relacionados con el empleo pleno y productivo y el trabajo decente para todas las personas.

- Objetivo 10: sobre la inclusión social, económica y política de todas las personas.

- Objetivo 11: señalando la necesidad de generar un sistema de transporte seguro, asequibles, accesibles y sostenibles para todos. El acceso universal a zonas verdes y espacios públicos seguros, inclusivos y accesibles.

- Objetivo 17: relacionado con el aumento de datos oportunos, fiables y de gran calidad desglosados por discapacidad.

\section{Marco legal de las personas con discapacidad en Nicaragua}

Respecto a la discapacidad, González (2012), plantea que ha sido abordada desde diversos puntos de vista, ordinariamente desde la perspectiva clínica de rehabilitación y diagnóstico. Según la Organización Mundial de la Salud -OMS- la población con discapacidad en el mundo asciende a más de 650 millones de personas. Todas comparten un común denominador: "siguen formando parte de los grupos más marginados en todas las sociedades" (p. 12). Por su parte, Nicaragua no está exenta de esta realidad. Las personas con discapacidad de todas las edades enfrentan serias limitaciones en el disfrute de sus derechos humanos más fundamentales de educación, salud, vivienda, trabajo digno, entre otras.

La Constitución Política de Nicaragua (CPN, 2014) es el principal documento nacional en donde se protege e informa sobre los derechos y obligaciones, también en materia referida a los derechos de las PCD, pues es necesario reconocer la obligación del Estado y de la sociedad en el cumplimiento de los mismos. Los principales artículos, hacen referencia a los programas estatales para discapacitados víctimas de guerra (Arto.
56); a su protección, recuperación de su salud (Arto. 59) y la rehabilitación física, psicosocial, así como profesional para su ubicación laboral (Arto. 62). Desde la visión de Samaniego (2009), la CPN también alude a la promoción de las PCD en cuanto a la Garantía de la educación como derecho social (Arto. 58); La creación de centros específicos (Arto. 76).

Por otra parte, en Nicaragua también se ha perfeccionado el marco legal para las $P C D$, con la creación, primero, de la Ley 202 de 1995 y su reglamento, derogados luego por la Ley 763 de 2011 y su reglamento de 2014. De acuerdo con Tobías (2013), esta nueva ley llena vacíos en cuanto a: acceso a infraestructuras, transporte, información y comunicación; Creación de los derechos civiles y políticos; Restitución del derecho a organizarse o participar en organizaciones y asociaciones que les representen tanto nacional como internacional; hincapié en el carácter coercitivo de la ley de discapacidad; Creación de la secretaría de promoción y articulación de los derechos de las personas con discapacidad y el consejo de promoción y aplicación de los derechos de las personas con discapacidad como órganos de organización, promoción, protección y aplicación de los derechos de las personas con discapacidad. No obstante, urge la aprobación de presupuesto para la aplicación de la ley (p. 64).

\section{La educación superior en Nicaragua y la generación de políticas públicas}

En este ensayo se plantea la importancia de la definición de políticas de extensión e inclusión de las PCD en las Instituciones de Educación Superior (IES). Las políticas públicas deben de generar una acción de cambio en el tiempo, con una visión a largo plazo donde todos participen en el consenso de esta. Una política pública debe definir las acciones de implementación y la gestión eficiente en su planificación y ejecución. Velázquez (2009), señala que una Política Pública (PP) es un proceso integrador de decisiones, acciones, inacciones, acuerdos e instrumentos, encaminado a solucionar o prevenir una situación definida como problemática en un ambiente determinado del cual se nutre y al cual pretende modificar o mantener (p.156).

Para que el sistema educativo nicaragüense pueda hacer efectivas las políticas públicas cuenta con un marco legal, basado en la Ley 89 , Ley de las Instituciones de Educación Superior (1990), Ley 582, Ley General de Educación (2006), la Ley 704, Ley Creadora del Sistema 
Nacional para el Aseguramiento de la Calidad de Educación Superior y Reguladora del Consejo Nacional de Evaluación y Acreditación (2011). Sin embargo, sólo la Ley General de Educación, en su Arto. 48, menciona al Consejo Nacional de Rectores, como el órgano encargado de políticas y quién será el responsable de definirlas para la educación superior del país. Y en su Arto. 49, a las universidades comunitarias de la Costa Caribe, quienes "podrán acompañar a los Consejos y Gobiernos Regionales Autónomos en el diseño e implementación de las políticas públicas de educación. Por otra parte, la misma Ley 582 establece en su Arto. 56 la Creación del Consejo Nacional de Educación y, además, en el Arto. 59 (inciso d), menciona que es parte de sus funciones la definición de las políticas educativas nacionales.

Sobre la base de este marco legal, las universidades públicas, en primer lugar, tienen la obligación de generar políticas, para lo cual una de las estrategias claves es la de la extensión universitaria. Para ello, el Glosario de Educación Superior de Nicaragua (CNEA, 2020), en su tercera edición, establece las definiciones de Extensión social, proyección o vinculación social, considerando la importancia de la existencia y pertinencia de políticas institucionales que la promuevan, los recursos, organización y capacidad instalada, los resultados de los procesos desarrollados, y los vínculos con la docencia e investigación. Siendo el profesional o académico un Extensionista que establece acciones de relación dialógica en el entorno universitario y hacia la sociedad asumiendo actitud de compromiso, responsabilidad y ética. Y la Política institucional, entendida como orientaciones, directrices y compromisos que rigen la actuación de la institución, dirigida a promover las acciones estratégicas y cumplir con las funciones, procedimientos y su respectivo control en la Institución de Educación Superior.

\section{La extensión universitaria y su papel de visibilizador de problemas sociales}

A pesar de que la universidad como institución ha funcionado por unos 800 años, con sus inicios en europeos en Bolonia, París y Oxford; y en el contexto latinoamericano en 1538 que se funda la Universidad Santo Domingo, en plena época de la conquista y colonización española, el tema de la extensión universitaria se remonta a la primera mitad del siglo XIX, siendo Simón Rodríguez, maestro de Simón Bolívar (el Libertador), quien empieza a utilizar el concepto de extensión cultural, que luego se denominaría extensión universitaria (Del Huerto Marimón, 2018, p. 38) aunque, como lo señalan González y Gonzáles (2018), los centros culturales y la divulgación de ideales progresistas, así como la integración de cholos y mestizos en las aulas aristocráticas, fue tildado de aventurero y utópico (p. 77).

Es con la llamada Reforma universitaria de Córdoba (Argentina), en el año 1918, cuando por vez primera se emplea en Latinoamérica el término extensión cultural universitaria como función consustancial de la universidad (Del Huerto Marimón, 2018, p. 38). El programa de esta reforma concibió la extensión universitaria como "el fortalecimiento de la función social de la Universidad, proyección al pueblo de la cultura universitaria y preocupación por los problemas nacionales" (Tünnerman, 2008, citado por Del Huerto Marimón, 2018, p. 39) la universidad, empieza entonces a comprenderse como institución a la que puede acceder personas de distintos estratos sociales y no solo ciertos privilegiados.

Definir qué es la extensión universitaria no es una tarea sencilla, por lo que se precisa retomar elementos conceptuales que en el tiempo han surgido (UNAN, 2018, p. 7-8), para poder identificar lo que ella significa, comprendiéndola como:

- Coparticipación entre docentes y estudiantes (Universidad Nacional de la Plata, Argentina, 1905)

- Incorporación del público en la obra docente de la universidad (Universidad de Oviedo, 1917)

- Comunicación dialógica, garante de la pertinencia y responsabilidad social (Freire, 1987)

- Función rectora en el vínculo entre universidad y sociedad (Encuentro binacional de directores de extensión, México, 1994)

- Proceso no formal de doble vía planificada de acuerdo a intereses y necesidades de la sociedad (Universidad Nacional de la Plata, Argentina, 2008)

- Función que orienta las líneas de investigación y planes de enseñanza, generando compromiso universitario (Universidad Nacional de la Plata, Argentina, 2009)

- Actividades desarrolladas por la universidad en pos de modificar la realidad social (Universidad Nacional del Litoral y la ULEU, Argentina, 2011) 
- Proceso de interacción, intercambio y comunidad (Universidad Federal de Santa Marta, Brasil, 2012)

- Responsabilidad social para extender su acción educativa hacia la comunidad nacional y fortalecer relaciones de cooperación e intercambio (UNAN, 2015)

- Aporte de la universidad a la sociedad cumpliendo roles de formación continua de la propia comunidad universitaria; de divulgación científica y de la diversidad cultural; en la transformación social y desarrollo comunitario; y en la transferencia tecnológica con visión estratégica de desarrollo (CNU, 2016)

De estos elementos se puede derivar una conceptualización de la extensión como un proceso universitario "tanto intramuros como extramuros" (Matus, Federico, comunicación personal, 14 de abril de 2021), donde fluye el conocimiento entre la universidad y la sociedad, en una relación dialógica entre personas que se reconocen de forma igualitaria y no discriminatoria, cuyos resultados enriquecen tanto al desarrollo de la sociedad como de la mejora de las funciones universitarias.

Aunque aún queda abierto el debate para aquellos que ven la extensión como difusión de cultura o conocimientos científicos, y la visión de la misma como extensión de servicios asistencias o académicos (Ruiz Lugo, 1992, en Fresán Orozco, 2004, p. 48), no queda duda que la extensión universitaria es una estrategia excelente para poder comprender mejor los problemas sociales y un instrumento posible que dé cauce a la generación de políticas públicas.

Para que la universidad sea visibilizadora de los problemas sociales, como el que acontece a las personas con discapacidad; es decir, es preciso que primero la universidad se haga visible a sí misma a través de las actividades de extensión. Como ejemplo, de la multiplicidad de modos y formas que pueden revestir estas actividades, se tienen: las investigaciones, las prácticas de familiarización, especialización y profesionalización de las distintas carreras; el voluntariado y servicio social comunitario; los programas educativos en el campo y la empresa; los programas ambientales, de salud, seguridad alimentaria y nutrición; los proyectos educativos; los programa de movilidad de docentes y estudiantes; los programas Interuniversitarios, entre otras (UNAN, 2015, p. 9).

En este proceso de visibilización, Fresán Orozco (2004), recuerda que la extensión universitaria aproxima tanto a los miembros de la comunidad educativa y los individuos y colectivos sociales en un camino comunicativo (de doble vía), que permita identificar con las necesidades sociales, se puedan hacer propuestas de solución (p.52), y permita la formación de individuos reflexivos y comprometidos con una sociedad menos injusta (p. 49), con la consecución de condiciones más propicias.

\section{Acciones acometidas desde la extensión en las universidades del CNU}

Según la consulta realizada en la encuesta empírica, aportada por siete funcionarios extensionistas representantes de las universidades miembros del CNU, el $28 \%$ de estos señala, que las principales discapacidades detectadas son de tipo auditivas y motoras; un $22 \%$ visuales; $17 \%$ de tipo verbal, y $6 \%$ de tipo múltiple. Por otra parte, se hizo consulta sobre el tipo de iniciativas formales, en ejecución o proyectadas, sobre protocolos de actuación, políticas de extensión y campañas de sensibilización, a fin de atender la situación de personas con discapacidad en estas universidades; el siguiente gráfico muestra el estado de estas actividades. 


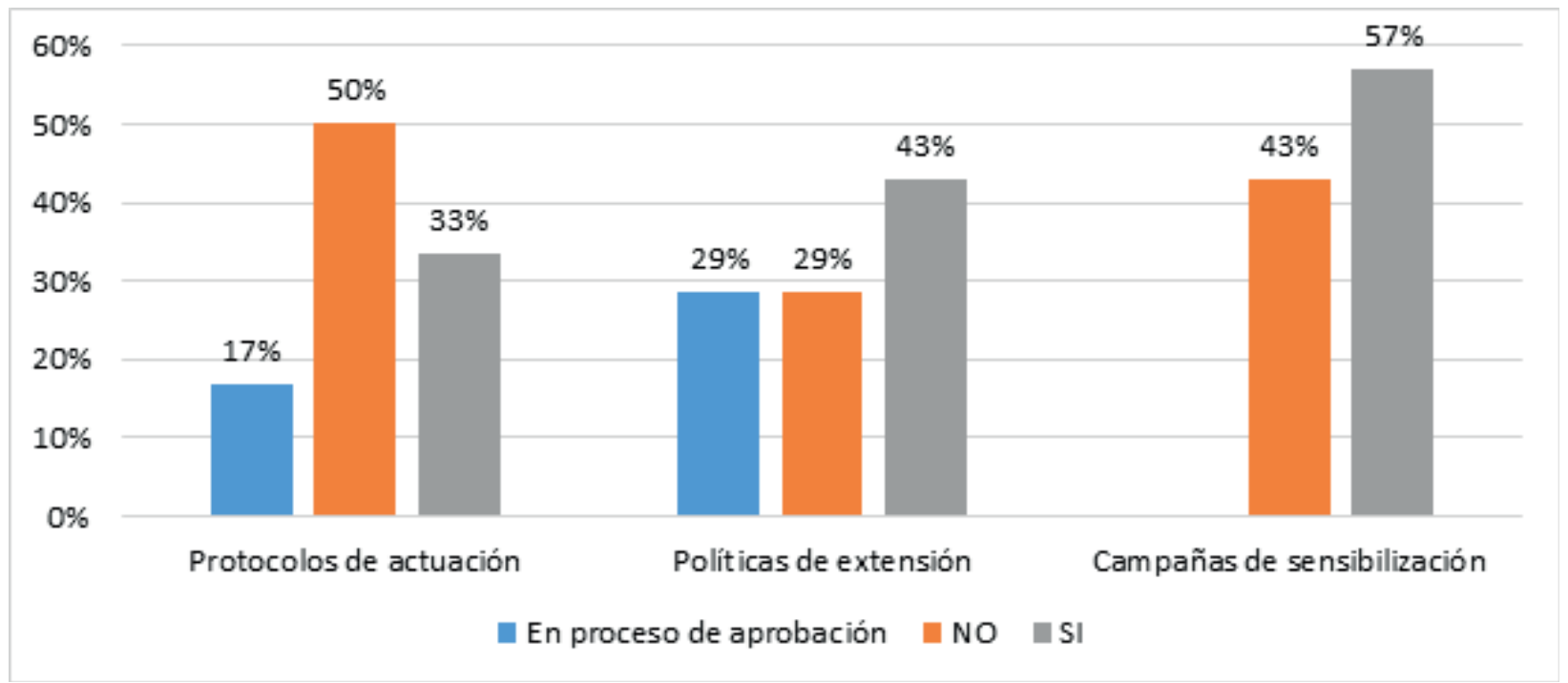

Gráfico 1. Acciones formales de atención a personas con discapacidad en universidades del CNU. Datos obtenidos de la consulta a extensionistas.

Según se observa, aún la mitad de las universidades no cuenta con protocolos de actuación, con el fin de atender a sus miembros con discapacidad. Menos de la mitad aún no cuenta con políticas de extensión aprobadas, en las que se considere este tipo de situación. $Y$ poco más de la mitad de universidades ha realizado algunas campañas de sensibilización en torno a las personas con discapacidad de la comunidad educativa.

Dentro de las experiencias relatadas por los extensionistas se destaca mencionar experiencias particulares sobre la inclusión de temas de discapacidad auditiva y visual dentro del currículum de algunas carreras; la creación de vídeos sobre la discapacidad, encuentros bilaterales con los estudiantes en condición de discapacidad, para conocer sus necesidades de movilización y su gestión educativa; la sistematización de la experiencia de inclusión socioeducativa de los estudiantes en condición de discapacidad; o la creación de una Norma Técnica Obligatoria de Accesibilidad, su instrumentación y una cartilla ilustrada para su divulgación popular.

Finalmente, los extensionistas recomiendan prestar atención a tres elementos cruciales, en torno a la discapacidad: a) La capacitación y sensibilización de la comunidad universitaria. b) Las mejoras en infraestructura que facilite el acceso al campus universitario. c) Destinar recursos económicos y humanos para la atención y medidas compensatorias para estos grupos tradicionalmente excluidos, segregados o inadvertidos.
Cruz, G. (2011). Programa Educativo "Cátedra de la Tierra Dr. Gonzalo Cruz Calderón”. Tegucigalpa, M.D.C.: Departamento de Ciencias Naturales, UPNFM.

Defensoría del Público de Servicios de Comunicación Audiovisual: https://defensadelpublico.gob.ar/ reflexiones-sobre-vejez-y-envejecimiento-enlos-medios-audiovisuales-junto-a-estudiantessecundarios/ y https://defensadelpublico.gob.ar/ tratamiento-responsable-sobre-las-personas-mayoresen-los-medios-audiovisuales/

Frávega, Adriana; Mársico Virginia. Representaciones y prácticas de recepción de las personas mayores frente a los discursos sociales en el marco de la Ley de Medios argentina. http://sedici.unlp.edu.ar/ handle/10915/44862.

Puca Molina, R. (2016). Los Adultos Mayores de Santa Cruz van a la Universidad. Extensión En Red, (7), 309316. Recuperado a partir de https://perio.unlp.edu.ar/ ojs/index.php/extensionenred/article/view/3370.

Ministerio de Comunicaciones. "Comunicación y envejecimiento. Ideas para una política". Dirección de Comunicación Social. Centro de Psicología Gerontológica - CEPSIGER. Bogotá, D. C., Colombia, julio 2002. 


\section{Conclusiones}

El tema de los derechos de las personas con discapacidad se ha convertido en uno de los grandes enfoques que a nivel internacional se ha promovido en los últimos tiempos. Sin embargo, el avance en esta materia, específicamente en los centros de educación superior es todavía incipiente. Se requiere que las universidades tomen mayores iniciativas, siendo la función de la Extensión un espacio privilegiado, tanto para visibilizar el problema, como para actuar sobre él.

No obstante, para que ello sea efectivo, se precisa la formalización de políticas de extensión que regulen y normen las prácticas, a fin de sistematizar los problemas, pasando de un ejercicio de simple voluntariedad, al desarrollo sistematizado de acciones sólidas que contribuyan a la generación de propuestas que puedan convertirse en políticas públicas en las agendas de los Estados. Y como ejemplo de algunas de las iniciativas, que deben formar parte primero de las políticas institucionales de la universidad, se pueden proponer las siguientes:

- Sensibilización y divulgación acerca de la discapacidad a los miembros de la comunidad universitaria.

- Acceso de una educación de calidad a los miembros de la comunidad universitaria con discapacidad.

- Creación de espacios físicos apropiados para los estudiantes con discapacidad.

- Promoción de investigaciones en torno a la discapacidad.

- Creación de indicadores de evaluación para la incorporación de personas con discapacidad a la educación superior.

- Definición de políticas que normen las actividades de extensión en las universidades, que le den un carácter más formal.

\section{Referencias bibliográficas}

- Constitución Política de Nicaragua (CPN 2014). Texto con reformas incorporadas a 2014. (Nicaragua)

- Convención internacional sobre los derechos de las personas con discapacidad, del 13 de diciembre de 2006. Consultado el 20 de abril de 2021. Recuperado de https://www.un.org/esa/socdev/ enable/documents/tccconvs.pdf

- Del Huerto Marimón, María Elena (2018). La Reforma universitaria de Córdoba: pionera en el legado latinoamericano de universidad popular. Revista Cubana de Educación Superior. (1) 37-47, consultado el 12 de abril de 2021. Recuperado de: http://scielo.sld.cu/pdf/rces/v37n1/rces03118.pdf

- Fresán Orozco, Magdalena (abril 2004). La extensión universitaria y la Universidad Pública. Reencuentro. (39) 47-54. Consultado el 12 de abril de 2021. Recuperado de: https://www.redalyc.org/ pdf/340/34003906.pdf

- González Sobalvarro, Julia (2012) Compilación de la legislación nicaragüense, en el tema de discapacidad. (Tesis nivel técnico). Escuela de Ciencias Psicológicas, Guatemala, Guatemala. (Inédito). Consultado el 10 de abril de 2021, recuperado de: http://www.repositorio.usac.edu. gt/12273/1/13\%20EE\%20\%28147\%29.pdf

- González, Gil y González, Mercedes (2018). A propósito de la Reforma de Córdoba: aproximación al origen y evolución de la extensión universitaria en América Latina y Cuba. Revista Cubana de Educación Superior. (1) 37-47, consultado el 12 de abril de 2021. Recuperado de: http://scielo.sld.cu/ pdf/rces/v37n1/rces06118.pdf

- Ley 202 de 1995. Ley de prevención, rehabilitación y equiparación de oportunidades para las personas con discapacidad. 23 de Agosto de 1995

- Ley 582 de 2006. Ley General de Educación. 22 de marzo del 2006.

- Ley 704 de 2011. Ley Creadora del Sistema Nacional para el Aseguramiento de la Calidad de Educación Superior y Reguladora del Consejo Nacional de Evaluación y Acreditación. 2 de septiembre del 2011. 
- Ley 763 de 2011. Ley de los derechos de las personas con discapacidad. 13 de abril del 2011.

- Ley 89 de 1990. Ley de las Instituciones de Educación Superior. 5 de abril de 1990.

- Naciones Unidas. Objetivos de Desarrollo Sostenible. Consultado el 20 de abril de 2020. Recuperado de: https://www.un.org/ sustainabledevelopment/es/objetivos-dedesarrollo-sostenible/

- Real Academia Española. Diccionario de la lengua española, $23^{\text {a }}$ ed., Consultada el 13 de mayo 2021. [versión 23.4 en línea]. https://dle.rae.es

- Reglamento a la ley de los derechos de las personas con discapacidad. 26 Febrero del 2014. Decreto 11-2014.

- Samaniego de García, P. S. (Dir.) (2009). Personas con discapacidad y acceso a servicios educativos en Latinoamérica. Ecuador: Grupo Editorial Cinca. https://sid-inico.usal.es/idocs/F8/FDO20981/ cermi_39.pdf

- Tobías Molina, Glenda (2013). Análisis comparativo de la Ley 202, Ley de Prevención, Rehabilitación y Equiparación de Oportunidades para las personas con Discapacidad en Nicaragua y Ley 763 , ley de los Derechos de las Personas con discapacidad en Nicaragua. (Monografía). Universidad Centroamericana-UCA. Managua, Nicaragua.

- Universidad Nacional Autónoma de Nicaragua UNAN (2018). Política de extensión y proyección universitaria 2017-2019 con su reforma. Dirección de Extensión Universitaria. Managua, Nicaragua. Recuperado de. https://direx.unan.edu.ni/ wp-content/uploads/2020/11/POLITICA-DEEXTENSIÓN-Y-PROYECCIÓN-UNIVERSITARIA-20172019-CON-SU-REFORMA.pdf

- Velásquez Gavilanes, Raúl (2009). Hacia una nueva definición del concepto "política pública". Desafíos. (20) 149-187 